\title{
Subaxial cervical spine trauma classification: the Subaxial Injury Classification system and case examples
}

\author{
Alpesh A. Patel, M.D. ${ }^{1}$ Andrew Dailey, M.D. ${ }^{2}$ Darrel S. Brodke, M.D. ${ }^{1}$ \\ Michael Daubs, M.D., ${ }^{1}$ Paul A. Anderson, M.D., ${ }^{3}$ R. John Hurlbert, M.D. ${ }^{4}$ \\ Alexander R. Vacccaro, M.D., Ph.D., ${ }^{5}$ and the Spine Trauma Study Group \\ Departments of ${ }^{1}$ Orthopaedics and ${ }^{2}$ Neurosurgery, University of Utah School of Medicine, Salt Lake City, \\ Utah; ${ }^{3}$ Department of Orthopedics and Rehabilitation Medicine, University of Wisconsin, Madison, \\ Wisconsin; ${ }^{4}$ University of Calgary Spine Program, Calgary, Alberta, Canada; and ${ }^{5}$ Department of \\ Orthopaedic Surgery, Thomas Jefferson University, Philadelphia, Pennsylvania
}

\begin{abstract}
Object. The authors review a novel subaxial cervical trauma classification system and demonstrate its application through a series of cervical trauma cases.

Methods. The Spine Trauma Study Group collaborated to create the Subaxial Injury Classification (SLIC) and Severity score. The SLIC system is reviewed and is applied to 3 cases of subaxial cervical trauma.

Results. The SLIC system identifies 3 major injury characteristics to describe subaxial cervical injuries: injury morphology, discoligamentous complex integrity, and neurological status. Minor injury characteristics include injury level and osseous fractures. Each major characteristic is assigned a numerical score based upon injury severity. The sum of these scores constitutes the injury severity score.

Conclusions. By addressing both discoligamentous integrity and neurological status, the SLIC system may overcome major limitations of earlier classification systems. The system incorporates a number of critical clinical variables - including neurological status, absent in earlier systems - and is simple to apply and may provide both diagnostic and prognostic information.(DOI: 10.3171/FOC.2008.25.11.E8)
\end{abstract}

KEY WORDS • cervical spine trauma $\quad$ classification
injury severity score $\quad \bullet \quad$ spinal cord injury

$\mathrm{T}$ HE treatment of subaxial cervical spine trauma is evolving. The development of cervical instrumentation and the proliferation of cervical fusion techniques have led to changes in the management of cervical trauma. Increasingly, cervical trauma is treated outside of tertiary or specialty care centers by physicians with varying levels of trauma care experience. These factors have lead to a significant variability in the management of cervical trauma. ${ }^{2}$

The variability in management of traumatic injuries is due, in part, to the lack of a widely accepted injury classification system. An ideal injury classification system, providing a common language, may improve patient care

\footnotetext{
Abbreviations used in this paper: DISH = diffuse idiopathic skeletal hyperostosis; DLC $=$ discoligamentous complex; SLIC $=$ Subaxial Injury Classification; VB = vertebral body.
}

by promoting standardized treatment strategies across medical centers and treating physicians. The ideal system must include key variables used by surgeons in medical decision making that influence clinical outcomes (for example, fracture level and morphological characteristics of injury, spinal alignment, neurological deficits, and expected long-term stability). Additionally, these variables should be appropriately weighted to account for their relative impact on medical decision making and clinical outcomes. The system would thus provide both descriptive and prognostic information.

A number of cervical injury classification systemsAllen et al., ${ }^{1}$ Harris et al., ${ }^{3}$ and White and Panjabi ${ }^{10}$ among others-have been described but none has demonstrated superior reliability or validity.1 ${ }^{11}$ Additionally, many of these systems do not take into account critical variables such as ligamentous and neurological injury. The SLIC system and Severity score has been developed in an attempt to address these limitations. ${ }^{7}$ The purpose of this 
paper is to review the SLIC system and demonstrate its application to 3 subaxial cervical trauma cases.

\section{The SLIC System and Severity Score}

The SLIC system and severity score identifies 3 major injury characteristics (injury morphology, DLC, and neurological status) with additional subgroups defined for each major descriptor (Table 1). Additional minor descriptors include spinal level, anatomical osseous injury descriptors, and injury confounders (ankylosing spondylitis, DISH, prior surgery, ongoing cord compression, and so forth). These confounders can influence surgical decision making. Each major characteristic is assigned a subscore; the subscores are then added to yield the severity score, which, in turn, can be used to guide treatment. The SLIC system is based upon the framework of the Thoracolumbar Injury Classification and Severity Score system, a validated thoracolumbar injury systemt..$^{5,8,9}$

\section{Injury Morphology}

The injury morphology defines the structural relationship of the VBs to each other in terms of anterior anatomical support, soft tissue structures, facet relationships, and overall alignment as determined by radiography.

Compression. Compression injuries are defined by a loss of height of the VB or disruption through the vertebral endplate. This includes traditional compression and burst fractures, as well as sagittal or coronal plane fractures of the VB.

Distraction. The distraction injury pattern is identified by anatomical dissociation of the spine through the vertical axis. Hyperextension injuries causing disruption of the anterior longitudinal ligament and subsequent widening of the anterior disc space are representative examples. Fractures of posterior elements (facets, laminae, and spinous processes) may be present. Bilateral perched facets, facet subluxation, or other kyphotic deformities of the spine, through a tensile failure of the ligamentous restraints, represent additional clinical examples of the distraction morphology.

Rotation/Translation. The rotation/translation injury is defined by horizontal displacement of one VB of the subaxial cervical spine with respect to another. Biomechanical work suggests that adequate thresholds for stability are $<3.5 \mathrm{~mm}$ of displacement or relative angulation of $<11^{\circ} .^{10}$ It is typified by unilateral and bilateral facet dislocations or fracture-dislocations, fracture separation of the lateral mass ("floating" lateral mass) with vertebral subluxation, and bilateral pedicle fractures with vertebral subluxation ("traumatic spondylolisthesis").

\section{Discoligamentous Complex}

The DLC defines the integrity of the intervertebral disc, anterior and posterior longitudinal ligaments, interspinous ligaments, facet capsules, and ligamentum flavum. This is a descriptor unique to the SLIC system and is divided into 3 categories: disrupted, indeterminate, and intact. Disruption of the DLC may be represented by
TABLE 1: The SLIC system

\begin{tabular}{|c|c|}
\hline Characteristic & Points \\
\hline \multicolumn{2}{|l|}{ morphology } \\
\hline no abnormality & 0 \\
\hline compression & 1 \\
\hline burst & $+1=2$ \\
\hline distraction (e.g., facet perch, hyperextension) & 3 \\
\hline $\begin{array}{l}\text { rotation/translation (e.g. facet dislocation, unstable } \\
\text { teardrop or advanced staged flexion compression injury) }\end{array}$ & 4 \\
\hline \multicolumn{2}{|l|}{ DLC } \\
\hline intact & 0 \\
\hline $\begin{array}{l}\text { indeterminate (e.g., isolated interspinous widening, MRI } \\
\text { signal change only) }\end{array}$ & 1 \\
\hline $\begin{array}{l}\text { disrupted (e.g., widening of anterior disc space, facet } \\
\text { perch or dislocation, kyphotic deformity) }\end{array}$ & 2 \\
\hline \multicolumn{2}{|l|}{ neurological status } \\
\hline intact & 0 \\
\hline root injury & 1 \\
\hline complete cord injury & 2 \\
\hline incomplete cord injury & 3 \\
\hline $\begin{array}{l}\text { ongoing cord compression (in setting of a neurologic } \\
\text { deficit) }\end{array}$ & +1 \\
\hline
\end{tabular}

abnormal facet alignment (articular apposition $<50 \%$ or diastasis $>2 \mathrm{~mm}$ through the facet joint), widening of the anterior disc space, translation or rotation of the VBs, or kyphotic alignment of the cervical spine. The additional finding of high signal intensity on T2-weighted fat-suppressed sagittal MR imaging seen through a disc involving the nucleus and anulus may infer disruption of the DLC. The "disrupted" designation should be assigned only when there is convincing evidence of DLC compromise.

Indeterminate injury may exist when radiographic disruption of the DLC is not otherwise obvious on radiographic or CT imaging but a hyperintense signal is found through either the disc or the posterior ligamentous regions on T2-weighted MR imaging images, suggesting edema and injury. Intact DLC is defined by normal spinal alignment in addition to normal disc space and ligamentous appearance.

\section{Neurological Status}

The neurological status of the patient is often the most influential component of medical decision making. Additionally, it can be inferred that neurological injury is a critical indicator of the degree of spinal column injury. The neurological status, another unique component of the SLIC system, is defined as 1 of the following: intact, root injury, complete cord injury, or incomplete cord injury. An additional modifier, continued cord compression, is described in the setting of either complete or incomplete spinal cord injury with ongoing spinal cord compression due to disc, bone, ligamentum, hematoma, or other structures. With translation or rotation injuries, assessment of cord compression should be made after attempted reduction of the injury. 


\section{Subaxial cervical spine trauma classification}

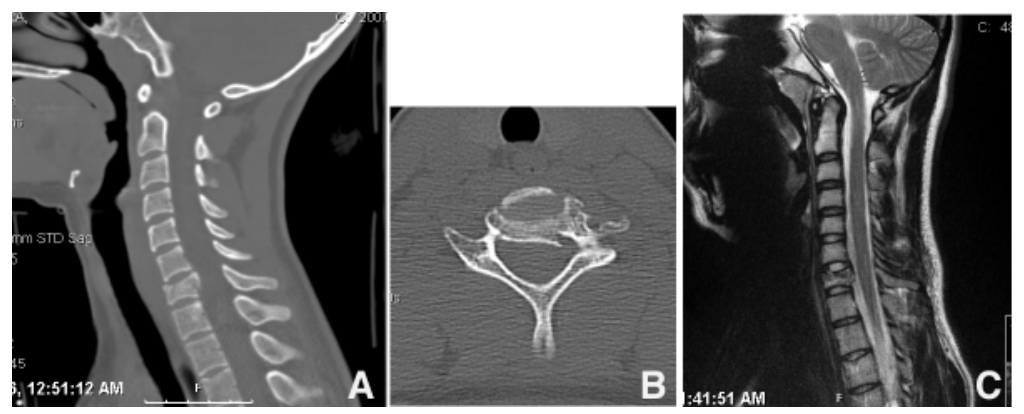

FIG. 1. Case 1. Injury resulting from a 30 -foot fall in a 17-year-old female patient. The patient presented with neck pain but there were no abnormal findings on neurological examination. A and B: Sagittal (A) and axial (B) CT images demonstrating a C-7 burst fracture. No focal kyphosis, posterior interspinous splaying, or facet subluxations are evident. C: Sagittal T2-weighted MR image showing normal signal intensity in the disc and both the anterior and posterior ligamentous structures.

\section{Application of the SLIC System}

A given subaxial cervical spine injury is classified using descriptors from each of the 3 major components of the SLIC system (morphology, DLC, and neurological status) as well the minor components (spinal level, anatomical osseous injury descriptors, and confounders) to form a comprehensive identification of the injury pattern.

Injuries are named according to the following 6 descriptors:

1. spinal level

2. injury morphology (major component)

3. osseous injury description (descriptors include fractures or dislocations of transverse processes, pedicles, endplates, superior and inferior articular processes, lateral masses, facet joints, laminae, and spinous processes).

4. status of the DLC (major component) with descriptors (for example, presence of a herniated nucleus pulposus)

5. neurological examination (major component)

6. confounders

Confounders describe preexisting cervical disease including: ankylosing spondylitis, DISH, osteoporosis, previous surgery, and degenerative disease. The presence of these confounders can influence not only the treatment decision (operative vs nonoperative) but also the surgical approach and the extent of surgical treatment. For example, the well-documented risks of neurological decompensation associated with concomitant ankylosing spondylitis or DISH may lead to operative treatment in patients with these conditions.

To estimate the severity of injury, a numerical value specific to each major component is generated. Injury patterns that are known to result in worse outcomes or require urgent surgical intervention (spinal instability, neurological injury) are weighted to receive greater point values. These 3 numbers, 1 from each component, are summed to provide an overall SLIC score. The resultant score can then be used to guide treatment of that particular injury. The higher the number of points assigned to a particular category, the more severe the injury and the more likely it is that a surgical procedure is indicated. In instances of multiple levels of cervical trauma, each level is treated as a separate injury, and SLIC scores are calculated independently for each level. If a single level has multiple injury patterns, only the most severe injury is considered for scoring. For example, if the injured level showed signs of both compression and rotation, morphology would be scored as rotation/translation. The descriptive identifiers and the point scores for each SLIC category are summarized in Table 1.

Surgical versus nonsurgical treatment is determined by a threshold value of the SLIC severity score. If the total score is $<4$ (that is, 1-3), nonoperative treatment is recommended. If the total is $\geq 5$, operative treatment is recommended. This treatment may consist of realignment, neurological decompression (if indicated), and stabilization. Cases with a total score of 4 may be treated operatively or nonoperatively.

\section{Illustrative Cases}

Three examples of cases of subaxial cervical spine injuries are presented to demonstrate the application of the SLIC system to describe the injury and to guide surgical decision making.

\section{Case 1}

This 17-year-old female patient presented after a 30 -foot-fall with severe neck pain. The results of her neurological examination were normal. Imaging studies revealed a C-7 burst fracture (Fig. 1). The injury was classified, according to the SLIC system, as a C-7 burst fracture with intact DLC in a neurologically intact patient.

This patient's injury was scored as follows: injury morphology_burst, 2 points; DLC - intact, 0 points; neurological status-intact, 0 points. Her total SLIC score was 2 , calling for nonoperative treatment.

\section{Case 2}

This a 53-year-old man presented with neck and unilateral (left) arm pain after a motorcycle accident. Physical examination revealed left biceps weakness and diminished light-touch and pin-prick sensation in the index finger. Imaging studies revealed anterior translation of C-5 on C-6 and articular facet fractures as well as 

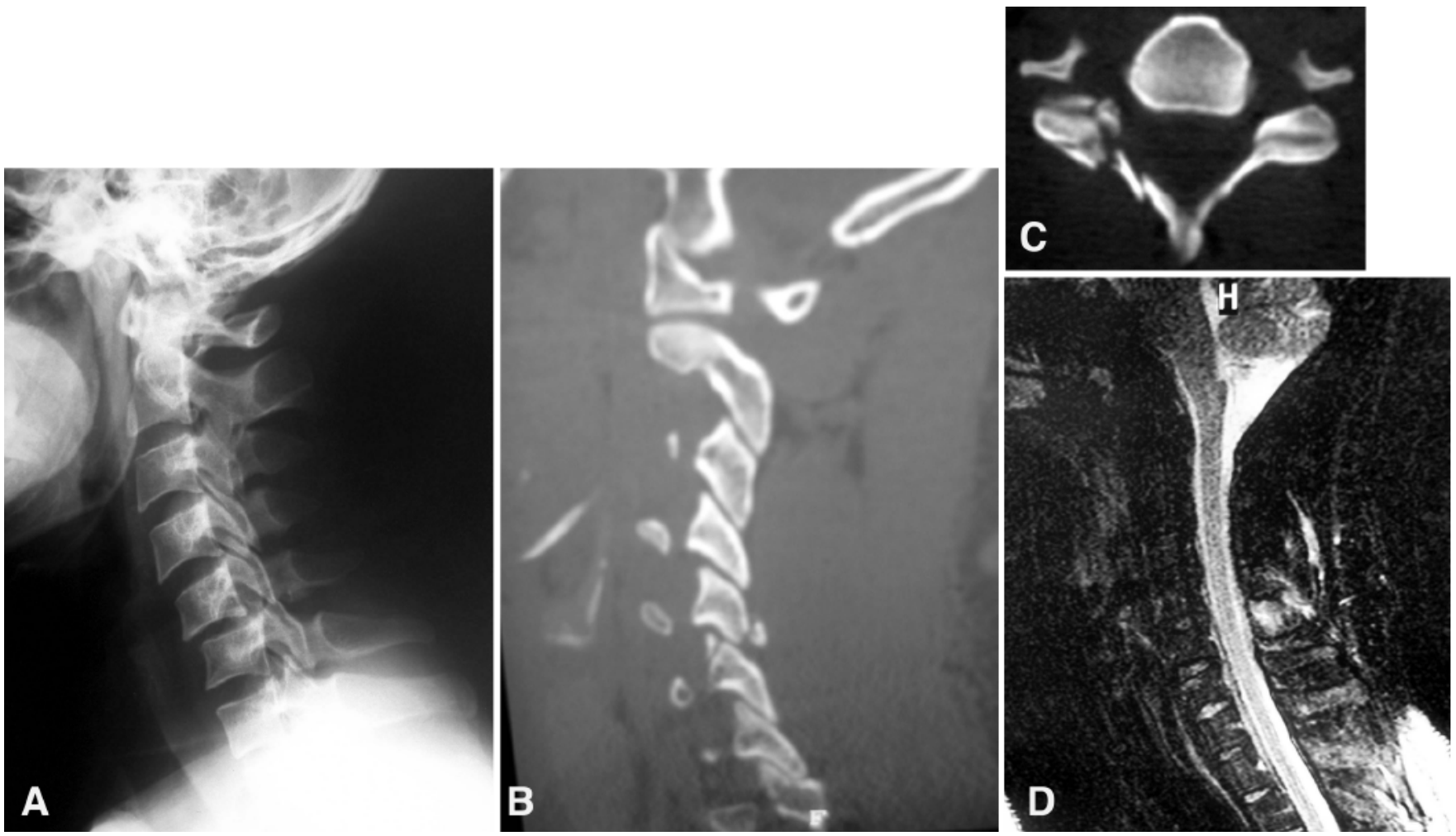

FIG. 2. Case 2. Injury resulting from a motor vehicle accident in a 53-year-old man. The patient presented with neck and left arm pain, biceps weakness, and numbness in the index finger. A: Lateral radiograph demonstrating anterior translation of C-5 on C-6. B and C: Parasagittal (B) and axial (C) CT images demonstrating C-5 inferior articular facet and C-6 superior articular facet fractures. D: Sagittal STIR sequence MR image revealing increased signal intensity in the posterior ligamentous structures.

demonstrating increased signal intensity in the posterior ligamentous structures (Fig. 2). The SLIC classification was -C5-6 translational injury with left facet fractures, a disrupted DLC, and a left C-6 nerve root injury.

This patient's injury was scored as follows: injury morphology_translational, 4 points; DLC-disrupted, 0 points; neurological status - root injury, 1 point. His total SLIC score was 7, indicating a need for operative treatment.

\section{Case 3}

This 32-year-old woman presented with a complete spinal cord injury (ASIA A) after a motor vehicle collision. Imaging studies revealed anterior dislocation of C-4 on C-5 and increased signal intensity in the intervertebral disc space and posterior ligamentous structures (Fig. 3). The SLIC classification was C4-5 translational injury with bilateral facet dislocations, a disrupted DLC, and a complete spinal cord injury.

This patient's injury was scored as follows: injury morphology_translational, 4 points; DLC-disrupted, 3 points; neurological status-complete SCI, 2 points. Her total SLIC score was 8 , indicating the need for operative treatment.

\section{Discussion}

Sir Frank Holdsworth used his experience with more than 2000 patients to create the first comprehensive classification system for spinal column injuries in $1970 .{ }^{4}$ In his landmark paper, Dr. Holdsworth was the first to discuss the importance of the posterior ligamentous complex in determining stability. Subsequently, 2 classification systems were proposed to specifically address subaxial cervical spine injuries. Allen and Ferguson, ${ }^{1}$ in 1982, built off their encounters with 165 patients to develop a system based on mechanism of injury inferred by the recoil position of the spine on plain radiographs. In 1986, Harris et al. ${ }^{3}$ suggested modifying the Allen and Ferguson system by including rotational vectors in flexion and extension.

Despite the comprehensive nature of these systems, their ultimate use is limited because they are based on a presumed mechanism of injury. These systems compartmentalize injuries into a variety of anatomical fracture patterns with arbitrary descriptors. Due to this fundamental deficiency, the systems lack practicality and clinical relevance and the associated terminology has been ineffective in describing traumatic conditions of the subaxial spine.

With the SLIC system and Severity score we attempt to resolve the shortcomings of prior systems by focusing on a framework that is clinically relevant, easy to apply, reliable, and free of geographic or language biases. The SLIC system has demonstrated good-to-excellent interrater reliability (ICC 0.71) when compared with the Ferguson and Allen and the Harris column systems ( 0.53 and 


\section{Subaxial cervical spine trauma classification}

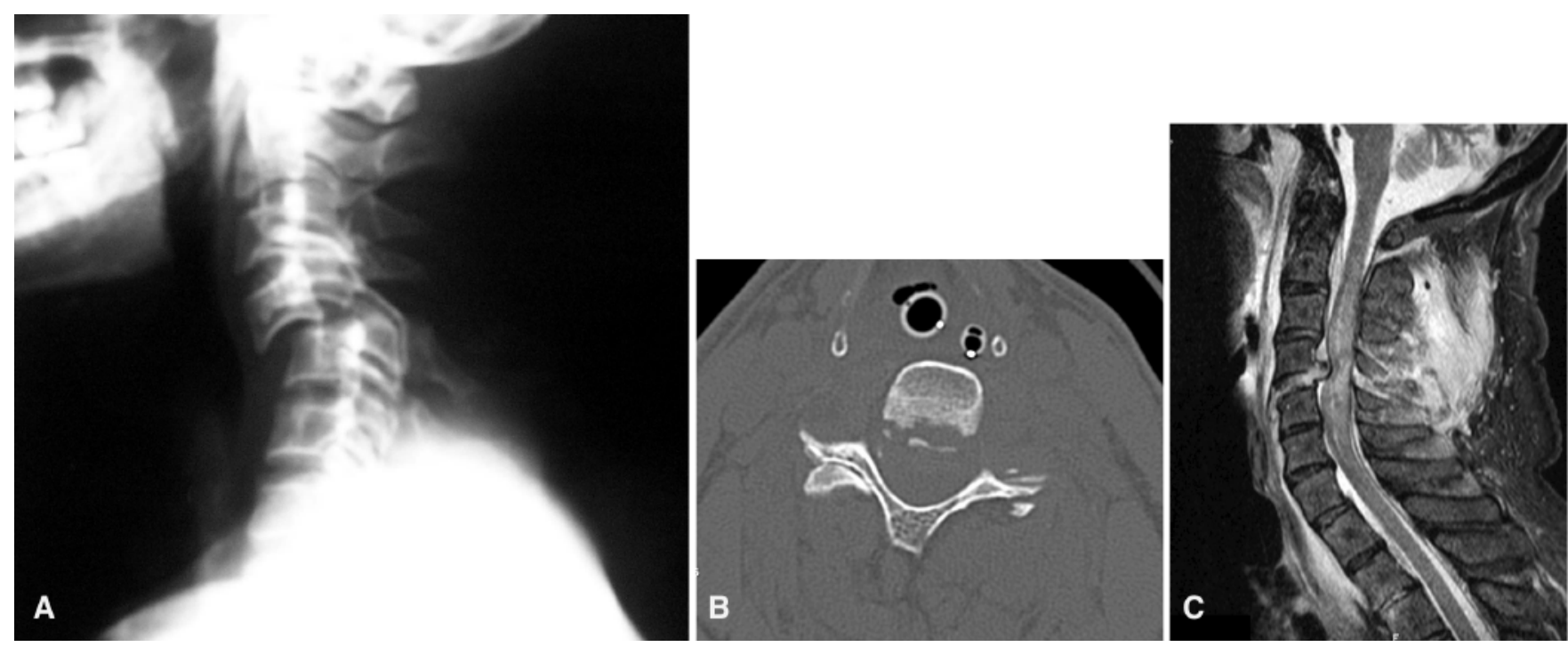

FIG. 3. Case 3. Injury resulting from an motor vehicle accident in a 32 year-old woman. The patient presented with neck pain and complete lower extremity paralysis. A: Lateral radiograph revealing anterior dislocation of C-4 on C-5. B: Axial CT scan demonstrating facet dislocations bilaterally. C: Sagittal STIR MR image revealing increased signal intensity in the intervertebral disc space as well as the posterior ligamentous structures.

0.42 , respectively). ${ }^{7}$ Intrarater reliability was also greater with the SLIC system than either of the previously described systems.

The SLIC system and Severity score is the first classification system to abandon an inferred mechanism of injury and, instead, base injury classification according on injury morphology. The subjective nature of previous inferred mechanistic classifications is reflected in poorer interrater reliability. ${ }^{6}$ This is also the first system to address both neurological status and DLC integrity, both powerful determinants of clinical treatment. Furthermore, this classification system is the first to suggest an optimal management strategy. Interrater agreement on the management of subaxial trauma has been reported at $74 \%$ using SLIC compared with 57-64\% with other systems; intrarater reliability using SLIC has been reported at $93 \%$ compared with $68-71 \%$.

The inclusion of the findings of neurological examination and advanced imaging technology into a classification system also presents potential difficulties and sources of bias. The SLIC system describes neurological injury as complete, incomplete, root level, or none. In the setting of spinal shock, the ability to distinguish complete from incomplete injuries may be difficult. The patient, in this scenario, may be best served by assuming an incomplete cord injury and treating appropriately. Additionally, neurological examination, despite its structured inclusion in the SLIC system, adds potentially subjective information into an otherwise objective classification.

The use of MR imaging in the assessment of the DLC is another potential shortcoming of the SLIC system. There is no evidence, to date, defining specificity and sensitivity of MR imaging in the diagnosis of discoligamentous disruption in the cervical spine. The use of the MR imaging alone in determining DLC status may, therefore, add error to the classification. For this reason, the SLIC system is intended to incorporate radiographic,
CT, and MR imaging findings in the assessment of DLC status. Individual interpretations, however, may still result in variations in SLIC scoring. This is reflected in the interrater reliability for each injury category, despite the overall good-to-excellent agreement with respect to treatment. Future studies, preferably prospective applications of the SLIC system and severity score, may further validate its use and its prognostic capabilities.

In summary, in this paper we have demonstrated the application of a novel subaxial cervical spine injury classification system and Severity score. We believe this system represents a significant improvement over current classification systems because it incorporates major clinical determinants for treatment and prognosis. These factors should enable this system to be widely accepted and highly effective in the discussion and treatment of subaxial cervical spine injuries. Nevertheless, there are certain limitations to the system, and incorporation of the system into future studies will allow for a better understanding of its validity and applicability.

\section{Disclosure}

This study was supported by the Spine Trauma Study Group and funded by an educational/research grant from Medtronic Sofamor Danek.

\section{References}

1. Allen BL Jr, Ferguson RL, Lehmann T: A mechanistic classification of closed, indirect fractures and dislocations of the lower cervical spine. Spine 7:1-27, 1982

2. Glaser JA, Jaworski BA, Cuddy BG, et al: Variation in surgical opinion regarding management of selected cervical spine injuries. A preliminary study. Spine 23:975-982, 1998

3. Harris JH, Edeiken-Monroe B, Kopansiky DR: A practical classification of acute cervical spine injuries. Orthop Clin North Am 17:15-30, 1986

4. Holdsworth F: Fractures, dislocations, and fracture-dislo- 


\section{A. A. Patel et al.}

cations of the spine. J Bone Joint Surg Am 52:1534-1551, 1970

5. Patel AA, Vaccaro AR, Albert TJ, et al: The adoption of a new classification system: time-dependent variation in interobserver reliability of the thoracolumbar injury severity score classification system. Spine 32:E105-E110, 2007

6. Schweitzer KM Jr, Vaccaro AR, Lee JY, et al: Confusion regarding mechanisms of injury in the setting of thoracolumbar spinal trauma: a survey of The Spine Trauma Study Group (STSG). J Spinal Disord Tech 19:528-530, 2006

7. Vaccaro AR, Hulbert RJ, Patel AA, et al: The subaxial cervical spine injury classification system: a novel approach to recognize the importance of morphology, neurology, and integrity of the disco-ligamentous complex. Spine 32:2365-2374, 2007

8. Vaccaro AR, Lehman RA Jr, Hurlbert RJ, et al: A new classification of thoracolumbar injuries: the importance of injury morphology, the integrity of the posterior ligamentous complex, and neurologic status. Spine 30:2325-2333, 2005
9. Whang PG, Vaccaro AR, Poelstra KA, et al: The influence of fracture mechanism and morphology on the reliability and validity of two novel thoracolumbar injury classification systems. Spine 32:791-795, 2007

10. White AA, Panjabi MM: Physical Properties and Functional Biomechanics of the Spine, in White A, Panjabi M (eds): Clinical Biomechanics of the Spine, ed 2. Philadelphia: Lippincott Williams \& Wilkens, 1990, p 22

11. Wood KB, Khanna G, Vaccaro AR, et al: Assessment of two thoracolumbar fracture classification systems as used by multiple surgeons. J Bone Joint Surg Am 87:1423-1429, 2005

Manuscript submitted July 10, 2008.

Accepted September 17, 2008.

Address correspondence to: Alpesh A. Patel, M.D., Department of Orthopaedic Surgery, University of Utah, Salt Lake City, Utah 84108.email: alpesh.patel@hsc.utah.edu. 